\title{
Rapid Distortion Theory and the structure of turbulence
}

\section{Julian Hunt Nicholas Kevlahan}

\begin{abstract}
This is a review of how, when linear distortions are applied to turbulent velocity fields, certain changes to some or all components of the turbulence can be calculated using linear theory. Important examples of such distortions are mean and random straining motions, body forces, interactions with other flows (eg. waves). This theory is usually known as Rapid Distortion Theory (RDT) because it is valid for all kinds of rapidly changing turbulent flows (RCT), when the distortion is applied for a time (defined in a Lagrangian frame) $T_{D}$ that is short compared to the 'turn-over' or decorrelation time scales $T_{L}$ or $\tau_{D}(k)$ of the energy containing eddies or smaller eddies of scale $k^{-1}$, respectively. However, for certain kinds of distortion the theory is also applicable to slowly changing turbulence (SCT) where $T_{D}$ is of the order or greater than $T_{L}$.

New insight about the structure of slowly changing turbulence has been derived from RDT by considering different strain rates, initial conditions and time scales. RDT calculations show that in shear flows, whatever the initial form of the energy spectrum $E(k)$ (provided it decreases with wavenumber $k$ faster than $k^{-2}$ ) or of the anisotropy, for a long enough period of strain, $E(k)$ always tends to the limiting form where it is proportional to $k^{-2}$ for the small scales. Other statistical properties, such as ratios of Reynolds stresses, are also insensitive to the initial conditions. By contrast RDT shows how turbulent flows without mean strain or with irrotational strain are significantly more sensitive to initial and external conditions. These conclusions are consistent with those drawn from Direct Numerical Simulations (DNS) and experiments at moderate Reynolds numbers.

The eddy structure of small scale turbulence can be studied by calculating the distortion of random Fourier components in a velocity field caused by different kinds of large scale straining motions. This method is used here in conjunction with an analysis of how the different types of straining motions in different regions of the small-scale turbulence are affected by the distortion. Interestingly, linear analysis shows that the vorticity vector tends to become aligned with the middle eigenvector of the rate of strain tensor, which is consistent with DNS for turbulent flows having a continuous spectrum.

At sufficiently high values of the Reynolds number, even in homogeneous and inhomogeneous distorted turbulence (mean straining flows, body forces and boundaries), the nonlinear processes act over a wide enough spectrum to ensure that at small scales
\end{abstract}


the energy spectrum $E(k)$ has an approximately universal form (as proposed by Kolmogorov). However, at the same time different components of the spectrum have an anisotropic structure. This can be estimated by applying RDT to each wavenumber component of the spectrum and taking the time of distortion to be approximately equal to the turnover time $t(k)$ appropriate to the value of $k$.

\section{Introduction}

Turbulence is a recognisable state of nature but it has no rigid definition; it is rather like certain diseases which are defined by a collection of symptoms called a syndrome. In the case of turbulence these 'symptoms' include randomness with a finite probability density function, strong vorticity, a complex highly three-dimensional velocity field, motion over a large and continuous range of length scales, and greatly increased effective values of viscosity and diffusivity. Many 'chaotic' flows, such as particular kinds of thermal convection, have some, but not all, of these 'symptoms'. The transition to turbulence only occurs when the Reynolds number (which is the ratio of inertial to viscous forces) or, in some cases the imposed disturbance, exceeds certain thresholds.

The conclusion from much recent research is that turbulence, even on the smallest scales, does not have a general statistical structure that is quite independent of initial and boundary conditions. Only for certain aspects of turbulence and in certain classes of flow does it appear that the eddy structure and statistical properties are insensitive to external conditions and large-scale motion. For example, Kolmogorov's inertial range theory is a good first approximation for the two-point second moments of the small scales and spectra in all turbulent flows at sufficiently high Reynolds number, but the higher order moments certainly do not have a universal structure (Hunt \& Vassilicos 1991, Frisch 1991). The intermediate or energy containing scales of turbulence and the Reynolds shear stresses do not generally have a universal structure, but in a wide class of shear flows similar eddy structures and statistical properties are found for these scales of motion (Townsend 1975, Hussain 1986). However, even in these flows, the largest scales that span the shear flow (such as wakes and jets) are quite specific to the particular mean flow profile and the history of the turbulence (Bevilaqua \& Lykoudis 1978, Mungal \& Hollingsworth; see review by Hunt 1992).

Flow visualisation and, more recently, computational studies have shown that within most turbulent velocity fields there are large eddy 'structures', within which the local distribution of velocity persists over time and space, and which have a repeatable flow pattern. These 'eddies' can be defined mathematically in a number of different ways, but most criteria for different types of eddies are based on a local strain rate or local variation of velocity (Wray \& Hunt 1990, Adrian \& Moin 1988, Mumford 1982). The structures of interest range in scale from very small scale vortical eddies that may be present in almost all high Reynolds number flows (eg. Hunt \& Vassilicos 1991) to large scale 'coherent structures' that span the entire width of shear flows and have characteristics peculiar to those flows (Hussain 1985).

Both quantitative models and qualitative analyses have shown how the salient features of the dynamics and kinematics of the whole flow, both instantaneously and statistically, can be explained in terms of the local dynamics and kinematics of these 
eddies (eg. Falco 1991, Hunt 1992, Townsend 1956). This approach is a more physically based and scientifically more fruitful method for studying the essential nonlinear and multi-scale dynamics of turbulence than that of developing complex statistical models based on uncertain mathematical approximation and hypotheses that are difficult to justify by physical arguments.

The primary aims of this review are to show how analyses of the linearised equations of motion, in the framework of Rapid Distortion Theory (RDT), can be used to explain some of the significant kinematical and dynamical aspects of the statistical properties and eddy structures in turbulence. (The aspects explained in this paper are briefly described in the summary.) Recent reviews have been written by Cambon 1992 and Hunt 1992b.

RDT uses linearised equations to describe the changes to a given velocity field $\mathbf{u}_{0}(\mathbf{x}, t)$ when it is subject to a distortion. Usually the given or initial velocity field is defined in terms of Fourier or other appropriate series, where amplitudes and phases are random. Then the problem reduces to calculating the 'transfer function' $M_{\text {in }}$ which determines how the amplitudes and phase of each component changes during the distortion. Once the transfer function is known (as in linear control theory) it is straight forward to calculate how the same distortion affects a wide range of initial turbulent velocity fields. For example some kinds of distortion are highly sensitive to the variation in initial conditions and others are not; the implications of this are discussed later.

Note that, although the methods of RDT are more general than those of linear hydrodynamic stability theory (HST), in fact these two types of calculation using the linearised solution of the equations of motion, are aimed at exploring quite different phenomena. Most HST calculations focus on solutions that grow exponentially in space and/or time, ie. solutions of the form $\mathbf{u} \propto \exp (-i(\alpha x+\sigma t))$, where the imaginary parts of $\alpha$ and $\sigma$ are positive. Experimentally, these are found to correspond to large-scale modes that span the whole mean flow; these are the modes that initially grow and determine the transition to turbulence. In a fully turbulent flow, the linearised modes of HST where $\operatorname{Im} \sigma=0$ (considered to be perturbations on the mean flow ignoring the fluctuations) correspond approximately to the largest scale eddy motion (Barcilon et al. 1974). RDT calculations may grow exponentially (as shown in $\S 3$ ), but usually they are applied to flows or parts of flows, or scales of eddy motion, where the disturbances do not grow exponentially fast (eg. Townsend 1976).

The idea of using linearised equations to describe how an initially prescribed velocity field is distorted by a shear or a straining flow began with Kelvin (1887). (His solution can be generalised to the nonlinear case Craik \& Criminale 1985.) The same mathematical formalism was first applied to distortions to turbulence by Taylor (1935) and was subsequently developed by Batchelor (1953), Batchelor \& Proudman (1954) and Townsend (1976). The original application of RDT was to calculate second order statistics such as changes in the intensities, correlations and (longitudinal one-dimensional) energy spectra resulting from the homogeneous distortion of homogeneous isotropic turbulence. The predictions of RDT were found to be satisfactory when compared to experimental results from a wind tunnel with a sudden contraction (eg. Tucker \& Reynolds 1968). 
RDT has since been extended and applied to other flows where the turbulence is undergoing inhomogeneous distortion, eg. around bluff bodies (Hunt et al. 1991), large scale flow in distorted ducts and non-isentropic compressible flow (Goldstein 1978), flow over hills and waves (Carruthers \& Hunt 1990), and flow in internal combustion engines (Reynolds 1980).

A less obvious extension of RDT has been its use as a way of analysing the structure of turbulence. In this case RDT is used to model individual realisations, instead of statistics averaged over many realizations. Recently Lee, Kim \& Moin (1990) have shown that an RDT shear calculation for slowly changing turbulence is able to produce the characteristic long, streaky structures observed in experiments on the turbulent boundary layer.

Another way in which RDT may be used to investigate the structure of turbulence is to divide the Fourier transform of the velocity into separate large-scale and small-scale parts and to model the interaction of the two scales as a rapid distortion of the small scales by the large scales (Kida \& Hunt 1989). By dividing the flow into a number of structure types and applying a variety of large scale distortions (corresponding to specific large scale structures) it is possible to see how various types of interaction affect the structure of the small scales and hence the properties of the turbulence. Dynamical quantities, such as vorticity production, can also be measured as a function of distortion to determine if the simple dynamics of this model can produce properties observed in experiments and Direct Numerical simulation (eg. the concentration of high intensity vorticity into thin filaments). The results of such an investigation will be presented in this paper.

The two-scale interaction model described above satisfies the usual condition of RDT, namely that it is valid for short times, because it considers local regions of the flow where it is assumed that each local small-scale region will only be subject to distortion by a given large-scale structure for a short time. One might also imagine trying to use RDT to analyse an entire flow over long times. The nonlinearity and independence from initial conditions which characterise turbulent flow make such a linear analysis seem unjustified. There is, however, the possibility that for some initial conditions and some types of strain the linear RDT solution reaches a steady 'eigensolution' that is independent of the initial conditions, or a 'statistical eigensolution' which is only weakly dependent on initial conditions, after averaging.

An example of such an 'eigenflow' was given by Hunt \& Carruthers (1990). They showed that for shear distortion of flows with energy spectra initially decreasing faster than $O\left(k^{-2}\right)$ the energy spectrum reaches a limiting form of $E(\chi) \propto \chi^{-2}$, where $\chi$ is the local (distorted) wavenumber. This means, for example, that a flow with initial energy spectrum $E(k) \propto \exp \left(-k^{2}\right)$ will change to a power law spectrum. This is exactly what is observed in Direct Numerical Simulations where the initial sharply peaked spectrum broadens to a steady power law spectrum after the turbulence has experienced significant straining by a shear flow.

Only turbulent flows (or components of such flows) that are independent of initial and boundary conditions can reasonably considered to be demonstrations of a 'universal nature of turbulence' (such as the small scale equilibrium theory of Kolmogorov 1941). Hence, the above example of eigensolutions in turbulent flows is important because 
of the evidence it provides as to what sort of turbulent flows may have universal characteristics. In this case, the mean shear imposes a certain statistical property and structure. This explains why many models of turbulence structure which neglect the effects of initial conditions are quite satisfactory for shear flows, but not for other kinds of distorted flows.

Although RDT is not itself a theory of turbulence, it can be used in conjunction with turbulence theories as guide to the modelling of dynamical terms. For example, in the closure theory EDQNM (Eddy Damped Quasi Normal Markovian) compressible RDT has recently been used to give the form of the pressure-dilatation term (Durbin \& Zeman 1992). These models may also be tested by comparing their results for rapid distortions with those predicted by RDT. Numerical experiments often produce surprising results, but cannot, of course, explain them. The other role of RDT in relation to models and simulations of turbulence is to help explain their results, eg. the alignment of vorticity and eigenvectors of the rate of strain which is presented in this paper.

\section{Formulation of RDT for homogeneous flows}

In this section we derive the equations for incompressible RDT and the criteria for their validity, starting from the Navier-Stokes equation. For a treatment of compressible RDT see, for example, Goldstein (1978). We focus here on homogeneous flows where the integral scale of the turbulence, $L_{x}$ is much less than the length scale $H$ over which the mean flow or the statistical properties of the turbulence vary. We consider the turbulence evolving in time; the results describe the distortion of turbulence along the mean streamlines in a statistically steady flow.

\subsection{Derivation of RDT equations}

Consider the turbulent velocity, $\mathbf{u}^{*}(\mathbf{x}, t)$, pressure, $p^{*}(\mathbf{x}, t)$, and vorticity, $\boldsymbol{\omega}^{*}(\mathbf{x}, t)$. Let $\mathbf{u}^{*}(\mathbf{x}, t), p^{*}(\mathbf{x}, t)$, and $\boldsymbol{\omega}^{*}(\mathbf{x}, t)$ be divided into mean and fluctuating components: $\mathbf{u}^{*}(\mathbf{x}, t)=\mathbf{U}(\mathbf{x}, t)+\mathbf{u}(\mathbf{x}, t), p^{*}(\mathbf{x}, t)=P(\mathbf{x}, t)+p(\mathbf{x}, t)$, and $\boldsymbol{\omega}^{*}(\mathbf{x}, t)=\boldsymbol{\Omega}(\mathbf{x}, t)+\boldsymbol{\omega}(\mathbf{x}, t)$, and substitute into the Navier-Stokes equations for incompressible flow,

$$
\begin{aligned}
\frac{\partial u_{i}}{\partial t}+U_{l} \frac{\partial u_{i}}{\partial x_{l}}+u_{l} \alpha_{i l}+u_{l} \frac{\partial u_{i}}{\partial x_{l}} & =-\frac{\partial p}{\partial x_{i}}+\nu \frac{\partial^{2} u_{i}}{\partial x_{l}^{2}}, \\
\frac{\partial u_{j}}{\partial x_{j}} & =0, \\
\omega_{i} & =\epsilon_{i j k} \frac{\partial u_{k}}{\partial x_{j}},
\end{aligned}
$$

where

$$
-\frac{\partial^{2} p}{\partial x_{l}^{2}}=2 \alpha_{l m} \frac{\partial u_{m}}{\partial x_{l}}+\frac{\partial u_{l}}{\partial x_{m}} \frac{\partial u_{m}}{\partial x_{l}}
$$


and where $\alpha_{i j}$ is the deformation tensor, $\partial U_{i} / \partial x_{j}$. If the input turbulence is homogeneous one may replace $u_{i}(\mathbf{x}, t)$ by its Fourier transform,

$$
\hat{u}_{i}(\boldsymbol{\chi}, t)=\frac{1}{(2 \pi)^{3}} \int u_{i}(\mathbf{x}, t) \exp [-i \boldsymbol{\chi} \cdot \mathbf{x}] \mathrm{d} \mathbf{x}
$$

and obtain equations for the evolution of the Fourier transform of the velocity,

$$
\begin{aligned}
\frac{\mathrm{d} \hat{u}_{i}(\boldsymbol{\chi}, t)}{\mathrm{d}} t= & -\chi^{2} \hat{u}_{i}(\boldsymbol{\chi}, t)-\alpha_{i l} \hat{u}_{l}(\boldsymbol{\chi}, t)+2 \frac{\chi_{i} \chi_{l}}{\chi^{2}} \alpha_{l m} \hat{u}_{m}(\boldsymbol{\chi}, t) \\
& -i \chi_{i} \mathbf{P}_{l m}(\boldsymbol{\chi}, t) \int \hat{u}_{l}\left(\boldsymbol{\chi}^{\prime}, t\right) \hat{u}_{m}\left(\boldsymbol{\chi}-\boldsymbol{\chi}^{\prime}, t\right) \mathrm{d} \boldsymbol{\chi}^{\prime},
\end{aligned}
$$

where $\mathbf{P}_{l m}(\boldsymbol{\chi}, t)$ is the projection operator (projects a vector into the plane perpendicular to $\chi)$,

$$
\mathbf{P}_{l m}(\chi, t)=\delta_{l m}-\frac{\chi_{l} \chi_{m}}{\chi^{2}}
$$

In order that equation (6) is homogeneous, it is necessary that the wave number $\chi$ changes according to the equation,

$$
\frac{\mathrm{d} \chi}{\mathrm{d} i} t=-\chi_{j} \alpha_{j i}
$$

This is equivalent to an equation for the conservation of wavefronts and shows that the motion of allowed wavenumbers in Fourier space is solenoidal. The Fourier transform of the vorticity, $\hat{\boldsymbol{\omega}}(\boldsymbol{\chi}, t)$, is related to $u_{i}(\boldsymbol{\chi}, t)$ by the equation,

$$
\hat{\omega}_{i}(\chi, t)=-i \epsilon_{i j k} \chi_{j} \hat{u}_{k}(\chi, t) .
$$

Note that in cases where the input velocity is not homogeneous the Fourier representation cannot be used, and so the velocity must be represented as a sum of a set of orthogonal basis functions calculated specifically for the initial flow. These functions are usually determined by the method of proper orthogonal decomposition (Lumley 1965). If the turbulence is homogeneous in only one or two directions the Fourier transform may be applied in those directions.

The first three terms in equation (6) are linear in the fluctuating component and represent changes in $\hat{u}_{i}(\boldsymbol{\chi}, t)$ due to viscous stresses and the interaction between the fluctuating and mean components. The last term on the right hand side of equation (6) represents changes due to interactions between fluctuating components of different wavenumbers. The rapid distortion approximation consists in assuming that this nonlinear term is negligible during the course of the distortion. Thus, the RDT approximation is equivalent to assuming that the fluctuating components do not interact with themselves and are only affected by the mean strain during the distortion (note that equation (8) for the evolution of the wavenumber is the same in both the linear and nonlinear cases). Expressed in terms of energy exchange, the RDT approximation takes into account transfer of energy from the mean flow to the fluctuating components, and redistribution of energy in $\boldsymbol{\chi}$-space, but not the nonlinear exchange of energy between Fourier amplitudes of different wavenumber. Usually the viscous term is ignored since it merely adds an exponential decay factor to the solutions. 
If the initial values of the Fourier transform of the velocity field are known one can calculate the distorted Fourier transforms of velocity and vorticity using RDT and then transform back to physical space to find the distorted velocity and vorticity fields. It is also possible to find directly a number of useful spectral quantities such as the energy spectrum tensor, $\Phi_{i j}(\boldsymbol{\chi}, t)$,

$$
\Phi_{i j}(\boldsymbol{\chi}, t)=\frac{1}{2} \overline{\left(\hat{u}_{i}^{*}(\boldsymbol{\chi}, t) \hat{u}_{j}(\boldsymbol{\chi}, t)+\hat{u}_{i}(\boldsymbol{\chi}, t) \hat{u}_{j}^{*}(\boldsymbol{\chi}, t)\right)} .
$$

Note that if the turbulence is initially isotropic the initial energy spectrum tensor may be calculated from the initial energy spectrum and initial wavenumbers,

$$
\Phi_{i j}(\mathbf{k}, t)=\frac{1}{4 \pi k^{4}}\left(\delta_{i j} k^{2}-k_{i} k_{j}\right) E(k, 0) .
$$

Where $\chi(t=0)=\mathbf{k}$.

The energy spectrum tensor is the three-dimensional Fourier transform of the correlation function, $R_{i j}(\mathbf{x}, \mathbf{x}+\mathbf{r} ; t)$,

$$
R_{i j}(\mathbf{x}, \mathbf{x}+\mathbf{r} ; t)=\overline{u_{i}(\mathbf{x}, t) u_{j}(\mathbf{x}+\mathbf{r}, t)},
$$

It is usually convenient to consider the integrated spectrum function, $E_{i j}(\chi, t)$,

$$
E_{i j}(\chi, t)=\frac{1}{2} \int_{|\chi|=\chi} \Phi_{i j}(\chi, t) \mathrm{d} S(\chi),
$$

where the integral is over a sphere of radius $\chi$ in wavenumber space, so that, $E_{i j}(\chi, t)$ gives the contribution to $\overline{u_{i} u_{j}}$ from length scales of magnitude $\chi^{-1}$. Thus, for example, $E_{11}$ gives the energy of the $x$-component of velocity as a function of time, and $E_{i i}(\chi, t)$ (or $E(\chi, t))$ gives the total energy contribution from length scale $\chi^{-1} . E(\chi, t)$ may be integrated once more to give the total energy of the distorted flow, $\overline{u_{i}^{2}(t)}$,

$$
\frac{1}{2} \overline{u_{i}^{2}(t)}=\int_{0}^{\infty} E(\chi, t) \mathrm{d} \chi
$$

Similar quantities may be defined and calculated for vorticity (Batchelor 1953, chap. 3).

Simple solutions to the RDT equations exist for pure rotational distortion, pure irrotational straining and pure shear (Townsend 1976). For example, Townsend's analytical solutions for simple shear (magnitudes of plane irrotational straining and rotational distortion are equal) of inviscid turbulence, $U_{1}=\alpha x_{3}$, are:

$$
\begin{aligned}
\hat{u}_{1}(\mathbf{k}, t) & =\frac{k^{2}}{k_{1}^{2}+k_{2}^{2}} Q_{1} \hat{u}_{3}(\mathbf{k}, 0)+\hat{u}_{1}(\mathbf{k}, 0) \\
\hat{u}_{2}(\mathbf{k}, t) & =\frac{k_{1} k_{2}}{k_{1}^{2}+k_{2}^{2}} Q_{2} \hat{u}_{3}(\mathbf{k}, 0)+\hat{u}_{2}(\mathbf{k}, 0) \\
\hat{u}_{3}(\mathbf{k}, t) & =\frac{k^{2}}{k^{2}-2 \beta k_{1} k_{3}+\beta^{2} k_{1}^{2}} \hat{u}_{3}(\mathbf{k}, 0)
\end{aligned}
$$


where $\mathbf{k}=\left(k_{1}, k_{2}, k_{3}\right)$ is the initial wavevector, $\chi=\left(k_{1}, k_{2}, k_{3}-\beta k_{1}\right)$ is the distorted wavevector, and,

$$
\begin{aligned}
Q_{1}= & -\frac{k_{2}^{2}}{k_{1}\left(k_{1}^{2}+k_{2}^{2}\right)^{1 / 2}} \arctan \left(\frac{\beta k_{1}\left(k_{1}^{2}+k_{2}^{2}\right)^{1 / 2}}{k^{2}-\beta k_{1} k_{3}}\right) \\
& +\frac{\beta k_{1}^{2}\left(k^{2}-2 k_{3}^{2}+\beta k_{1} k_{3}\right)}{k^{2}\left(k^{2}-2 \beta k_{1} k_{3}+\beta^{2} k_{1}^{2}\right)} \\
Q_{2}= & \frac{k^{2}}{k_{1}\left(k_{1}^{2}+k_{2}^{2}\right)^{1 / 2}} \arctan \left(\frac{\beta k_{1}\left(k_{1}^{2}+k_{2}^{2}\right)^{1 / 2}}{k^{2}-\beta k_{1} k_{3}}\right) \\
& +\frac{\beta\left(k^{2}-2 k_{3}^{2}+\beta k_{1} k_{3}\right)}{k^{2}-2 \beta k_{1} k_{3}+\beta^{2} k_{1}^{2}} \\
\beta= & \alpha t
\end{aligned}
$$

Note that for homogeneous three-dimensional distortions consisting of arbitrary proportions of irrotational and rotational straining over finite times even the linearised RDT equations cannot be solved in closed form for arbitrary initial conditions (Craik \& Criminale 1986), and so one must resort to asymptotic methods.

Thus, we have seen that RDT provides a straightforward method for calculating changes in turbulent velocity and vorticity fields and their second order moments given the initial velocity (or vorticity) field and the distorting field, specified in terms of a deformation tensor. The next question to be addressed is under which conditions the RDT linearisation is justified.

\subsection{Criteria for the RDT linearisation}

As suggested by its name, the usual criterion for the application of RDT is that the distortion takes place so rapidly that the inertial forces of the distorted turbulence have no effect, ie.,

$$
T_{D} \frac{u(l)}{l} \ll 1
$$

where $l$ is the length-scale of the distorted turbulence and $T_{D}$ is the time-scale of the distortion. Another criterion is that the magnitude of the nonlinear turbulent terms be small compared with the distorting terms,

$$
\frac{u^{2}(l) / l}{u(l) U / L_{D}} \ll 1,
$$

where $L_{D}$ is the length-scale of the distorting velocity field. The nonlinear terms will lead to significant transfer of vorticity into directions away from that of the maximum applied straining (linear analysis says that vorticity will only be transferred to the direction of maximum applied straining).

Thus, equation (22) must be modified to take into account the nonlinear rotation of vorticity if RDT is to be valid for all components of velocity and vorticity:

$$
\frac{u^{2}(l) / l}{\theta\left(T_{D}\right) u(l) U / L_{D}} \ll 1
$$


where $\theta\left(T_{D}\right)=\exp \left(\left(\lambda_{\min }-\lambda_{\max }\right) T_{D}\right)$ and $\lambda_{\min }$ and $\lambda_{\max }$ are the moduli of the minimum and maximum values of the principal strains of the deformation tensor. This means that if the strain is strong and isotropic the nonlinear terms may be neglected for all time (Batchelor 1955). The value of $\theta\left(T_{D}\right)$ will depend on the typical eddy structures making up the turbulent flow. Combining criteria (21) and (23) we have the general conditions on velocity, lengths and times for applying the RDT linearisation:

$$
\frac{u(l)}{l} \ll \max \left(\frac{U}{L_{D}} \theta\left(T_{D}\right), \frac{1}{T_{D}}\right)
$$

These conditions may be summarised as follows: RDT is always valid as long as either the strain rate is large enough (taking into account nonlinear rotation effects), or the period of the distortion, $T_{D}$, is short enough. Note that this does not imply that the time-scale of the large eddies must be less than the time-scale of the small eddies. In certain circumstances, considered below, RDT (despite its name) may actually be applied over long periods of time (ie. it may be used to analyse slowly changing turbulence) if the nonlinear term remains small compared to the linear terms.

The criteria derived above for the application of RDT to a turbulent velocity field all arise from an analysis of the turbulent Navier-Stokes equations in physical space, before the Fourier transform has been taken. These criteria are based on the relating acceleration to magnitudes of velocity by assuming that the motions are similar to oscillatory wave motion. They do not take into account the actual spatial structure of the turbulence.

An alternative new way of looking at the conditions under which RDT is valid is to retain the nonlinear terms and examine their magnitude in Fourier space. The nonlinear term in equation (6) is the nonlinear one and is seen to be responsible for the nonlinear exchange of energy between wavenumbers in spectral space, and is essentially the nonlinear transfer term, $T(\chi)$ in the energy evolution equation for a distorted turbulent flow,

$$
\begin{aligned}
\frac{\partial E(\chi)}{\partial t} & =-2 \nu \chi^{2} E(\chi)-2 \alpha_{i j} \int_{|\boldsymbol{\chi}|=\chi} \Phi_{i j}(\boldsymbol{\chi}) \mathrm{d} S(\boldsymbol{\chi}) \\
& +\alpha_{i j} \frac{\partial}{\partial \chi} \int_{|\boldsymbol{\chi}|=\chi} \frac{\chi_{i} \chi_{j}}{\chi} \Phi_{l l}(\boldsymbol{\chi}) \mathrm{d} S(\boldsymbol{\chi})-T(\chi)
\end{aligned}
$$

The first term on the right hand side is the decay of energy due to viscosity, the second is the transfer of energy from the distorting flow to the distorted flow, and the third is the redistribution of energy in Fourier space caused by the deformation of the distorted flow and $T(\chi)$ is the transfer of energy from all wavenumbers of magnitude less than $\chi$ to all wavenumbers of magnitude greater than $\chi$.

It is clear from inspection of the equations in Fourier space that the neglect of the nonlinear terms required by RDT is equivalent to assuming that during the distortion no energy is being transferred between length-scales except due to deformation of the velocity field. This means that an alternate RDT criterion based on the organisation of the distorted velocity field may be given: RDT may be applied for long times and for any level of strain as long as the transfers of energy between length-scales due to 
the distortion are much larger than the nonlinear transfer of energy, ie.,

$$
\overline{|T(\chi)|} \ll \min \left(\overline{2 \alpha_{i j} \int_{|\boldsymbol{\chi}|=\chi} \Phi_{i j}(\boldsymbol{\chi}) \mathrm{d} S(\boldsymbol{\chi})}, \overline{\alpha_{i j} \frac{\partial}{\partial \chi} \int_{|\boldsymbol{\chi}|=\chi} \frac{\chi_{i} \chi_{j}}{\chi} \Phi_{l l}(\boldsymbol{\chi}) \mathrm{d} S(\boldsymbol{\chi})}\right)
$$

Different turbulent flows will have different forms for $T(\chi)$ depending mainly on the type and distribution of structures they contain. Indeed for some special flows (eg. the symmetric 'error function' eddy, $u_{\theta}(r)=r \exp \left(-\frac{1}{2} \alpha^{2} r^{2}\right)$, or the potential spiral vortex, $\left.\mathbf{u}(r, \theta)=-\left(c r^{\frac{1}{\alpha}-1}\right) \hat{\mathbf{r}}-(c / r) \hat{\boldsymbol{\theta}}\right)$ it can be shown that $T(\chi)$ is identically zero. In that case the nonlinear inertial terms are initially zero and the RDT form of the equations is exact. However, in most subseqent distortions these terms will cease to be zero and the RDT solution will only be approximate. Thus the criteria for the validity of RDT should be based on the organisation of the velocity field, as well as its magnitude. Incidentally, this approach helps us understand the RDT approximation in terms of energy dynamics instead of inertial forces and time-scales. The organisational and energetic basis of this criterion makes it extremely useful when RDT is used as a way of exploring the structures of turbulence.

\subsection{Initial and boundary conditions in RDT}

An aspect of RDT that distinguishes it from models of turbulence such as EDQNM (Eddy Damped Quasi-Normal Markovian) is that the initial conditions may be nonstatistical. This would suggest that RDT is unsuitable for investigating characteristic structures of nonlinear turbulence, which because of its nonlinearity quickly becomes uncorrelated with its initial conditions and often reaches a 'universal' form. Models such as EDQNM are best suited for use with turbulent flows having a universal statistical form (eg. shear flows). An RDT analysis should be useful for other, non-universal, flows. RDT should also be valuable in analysing flow which vary slowly with time and are weakly dependent on initial conditions - these are characteristic 'eigensolutions' or 'statistical eigensolutions'. In such cases the form of the energy spectrum (though not its magnitude) reaches a statistically steady state if RDT is be applied over a long time. (This does not necessarily imply that it is valid over a long time though experimentally it is found that the predicted form of $E(k)$ is correct.) This is another example of an energy and organisationally based criterion for the application of RDT. An example of such an 'eigenflow' will be given in $\S 3.2$ below. Since the various types of turbulent flow show different degrees of correlation with initial conditions, the models of turbulence are not all equally effective at predicting their flow properties. Table 1 below compares the effectiveness of various models for different flows (defined by time scales) and indicates which flows can be treated using RDT. Table 1 shows that RDT gives better results than the 1-point eddy viscosity and second order Reynolds stress transport models for certain quantities in many types of flows, and is comparable to the more complex 2-point EDQNM closure model. This is perhaps surprising considering that RDT is a linear theory and was originally intended for flows with $T_{D} / T_{L} \ll 1$.

In RDT and in DNS the boundary conditions can be applied before any statistical averaging is performed. This contrasts with the Reynolds stress models where the boundary conditions can only be specified in terms of the moments or other statistical 


\begin{tabular}{|c|c|c|c|c|c|c|}
\hline \multirow[t]{2}{*}{ Property of flow } & \multirow{2}{*}{$\begin{array}{l}\text { Observations or num- } \\
\text { erical simulations }\end{array}$} & \multirow[b]{2}{*}{ Time scales } & \multicolumn{2}{|c|}{ 1-P models } & \multicolumn{2}{|c|}{$2-\mathrm{P}$ models } \\
\hline & & & EVM & 2RST & RDT & EDQNM \\
\hline \multirow[t]{2}{*}{ (i) $\frac{\overline{u_{i} u_{j}}}{K}, \frac{p \partial u_{i} / \partial x_{j}}{K S}$} & & $T_{L} S \leq 1$ & $\begin{array}{l}\mathrm{Y}(\mathrm{Sh}), \mathrm{N} \\
\mathrm{N}(\mathrm{NSh}), \mathrm{N}\end{array}$ & $\mathrm{Y}(\mathrm{G})$ & $\mathrm{Y}(\mathrm{P})$ & $Y(G)$ \\
\hline & & $T_{L} S \gg 1$ & $\mathrm{~N}$ & $\mathrm{Y}(\mathrm{P})$ & $\mathrm{Y}(\mathrm{G})$ & $\mathrm{Y}(\mathrm{G})$ \\
\hline \multirow{4}{*}{$\begin{array}{l}\text { (ii) Correct sensitivity of } \\
\text { property (i) to the } \\
\text { initial } E(k)\end{array}$} & None & $T_{D} / T_{L} \ll 1$ & $\mathrm{Y}$ & Y & $\mathrm{Y}$ & $\mathrm{Y}$ \\
\hline & Some & $T_{D} / T L \sim 1$ & $\mathrm{~N}$ & $\mathrm{~N}$ & $\mathrm{Y}$ & $\mathrm{Y}$ \\
\hline & None (Sh) & $T_{D} / T_{L} \gg 1$ & $\mathrm{Y}(\mathrm{Sh})$ & $\mathrm{Y}(\mathrm{Sh})$ & $\mathrm{Y}$ & $\mathrm{Y}$ \\
\hline & Some (NSh) & $T_{L} S \geq 1$ & $\mathrm{~N}(\mathrm{NSh})$ & $\mathrm{N}(\mathrm{NSh})$ & $\mathrm{Y}$ & $\mathrm{Y}$ \\
\hline \multirow{4}{*}{$\begin{array}{l}\text { (iii) Correct sensitivity of } \\
\text { property (i) to the initial } \\
\text { anisotropy } b_{i j}, c_{i j}\end{array}$} & Some & $T_{D} / T_{L} \ll 1$ & $\mathrm{~N}$ & Y (approx.) & $\mathrm{Y}$ & $\mathrm{Y}$ \\
\hline & Weak & $T_{D} / T_{L} \sim 1$ & $\mathrm{~N}$ & $\mathrm{Y}$ & $\mathrm{Y}(?)$ & $\mathrm{Y}$ \\
\hline & None (Sh) & $T_{D} / T_{L} \gg 1$ & $\mathrm{Y}(\mathrm{Sh})$ & $\mathrm{Y}(\mathrm{Sh})$ & $\mathrm{N}(\mathrm{Sh})$ & $\mathrm{N}$ \\
\hline & Some (NSh) & & N (NSh) & Y (NSh) & Y (NSh) & $\mathrm{Y}$ \\
\hline (iv) Correlation of $u_{i}(\mathbf{x})$ & Strong & $T_{D} / T_{L}<1$ & $\mathrm{~N}$ & $\mathrm{~N}$ & $Y(G)$ & $\mathrm{Y}(?)$ \\
\hline $\begin{array}{l}\text { with initial (or boundary) } \\
\text { turbulence }\end{array}$ & Weak & $T_{D} / T_{L} \geq 1$ & & & $\mathrm{Y}(\mathrm{P})$ & $\mathrm{Y}$ \\
\hline
\end{tabular}

Table 1: Comparative merits of 1-point (1-PM) and 2-point (2-PM) models for different flow properties and turbulent flows (defined by time-scales) in homogeneous strains with shear (Sh) and without shear (NSh) away from rigid boundaries or other interfaces. $T_{L}$ is the time-scale of the turbulence, $T_{D}$ is the imposed time-scale of the distortion, and $S$ is the applied strain rate, and $K$ is the kinetic energy per unit mass. EVM $=$ Eddy Viscosity Models; 2RST $=$ Second Order Reynolds Stress Transport; RDT $=$ Rapid Distortion Theory; EDQNM = Eddy Damped Quasi-Normal Markovian. Y $=$ Yes, can be computed or is correct in principle; $\mathrm{N}=\mathrm{No},(\mathrm{G})=$ Good Model in practice; $(\mathrm{P})=$ Poor Model in practice. (From Hunt 1992). 
quantities. This involves some serious assumptions near the boundary (Hunt 1992a). For the RDT or DNS computations on a rigid boundary layer of thickness $l_{\delta}$ for an eddy of length-scale $l$ at high Reynolds number $u(l) l / \nu \gg 1$ the boundary conditions are,

$$
\begin{array}{ccc}
n<l_{\delta} & \mathbf{u}=0 & \text { on } n=0 \\
n>l_{\delta} & \mathbf{u} \cdot \mathbf{n}=0 & \text { on } n=0
\end{array}
$$

where $n$ is the distance from the wall, and $l_{\delta}$ is the viscous layer generated on the wall by the eddy moving over wall. (For typical laboratory scale turbulence where $u l / \nu \sim \omega^{2}$, $l_{\delta} \sim\left(l_{\delta} \nu / u(l)\right)^{1 / 2}$.). Typically, if $n \gg 2 l$ the wall boundary condition is irrelevant for an eddy of scale $l$. This is why in shear boundary layers where $L_{x} \sim(1 / 2) n$, the direct effects of the wall on eddies is small, whereas in shear free boundary layers $L_{x} \sim n$, the direct effects are large.

\section{Turbulent structures and statistical models of tur- bulence}

\subsection{RDT and other methods of calculation}

In this section we discuss how RDT has been used in the investigation of turbulent structures and present some new results on structural and dynamical changes in turbulence undergoing arbitrary two-dimensional distortions. This distortion is considered as a model of some of the dynamics of the interaction between the large-scales and small-scales of turbulence. We also examine the phenomenon of turbulent 'eigenflows' which were discovered by Hunt \& Carruthers (1990) on the basis of an RDT analysis of turbulent shear flows. This result suggests why similar forms of spectra are found in turbulent shear flows at values of the Reynolds number $(R e)$ too small to produce the universal structure predicted by Kolmogorov's nonlinear cascade model. At these values of $R e$ RDT and experiments show that no universal form exists for unsheared turbulence, which explains the success of statistical turbulence models in predicting moments and a wide range of spectra in shear flows, but not in unsheared flows.

RDT was originally developed to predict changes in the statistics of rapidly distorted turbulent flows, its application to the analysis of structures in turbulence is new. However, it is also suited to the analysis of eddy structure of turbulence, which is not possible with purely statistical models such as eddy viscosity or $K-\epsilon$. The only requirements for the application of RDT are that one of the sets of criteria for the application of RDT be satisfied in order that the linearisation be valid. Even if the criteria are not met an RDT analysis may still be valuable since, by comparing the dynamics produced by the linear RDT theory with corresponding results from experiment or Direct $\mathrm{Nu}^{-}$ merical Simulation (DNS), the degree of dependence of particular dynamical quantities (such as vorticity production) on nonlinear processes may be gauged.

In order not to prejudice the result the exploration of the eddy structure of turbulence using RDT is to begin by considering an isotropic, homogeneous turbulent 
velocity field (either from experiment, Direct Numerical Simulation, or randomly generated with prescribed energy spectrum) and then applying to it a distortion typical of the flow to be examined (eg. apply a strong shear to investigate turbulent boundary layers). After the distortion has been applied the flow is analysed to look for any changes in the number and distribution of various structure types.

\subsection{RDT and statistical turbulent 'eigenflows'}

In this section we briefly review the results of Hunt \& Carruthers (1990) who used Townsend's (1976) RDT analysis of sheared turbulence to look for universal features in the spectra of the small-scale turbulence in shear flow.

Consider a uniform shear $\mathbf{U}=\left(\alpha x_{2}, 0,0\right)$ rapidly distorting an initially homogeneous and isotropic turbulent velocity field $\mathbf{u}$. The Fourier components of $\mathbf{u}$ will evolve as given by equations (15), (16), (17). The spectra will be expressed in terms of the local wavenumber, $\boldsymbol{\chi}$, (which is what is actually measured),

$$
\chi^{2}=k_{1}^{2}+\left(k_{2}-k_{1} \beta\right)^{2}+k_{3}^{2},
$$

where $\beta=\alpha t$. Since the input velocity field is assumed to be isotropic the components of $E_{i i}(\chi)$ may be calculated from equation (11),

$$
\begin{aligned}
E_{11}(\chi, t)= & \int_{|\boldsymbol{\chi}|=\chi} \frac{E(k, 0)}{4 \pi k^{2}}\left\{\frac{k^{2}}{\left(k_{1}^{2}+k_{3}^{2}\right)}(a+b)\left((a+b)-\frac{2 k_{1} k_{2}}{k^{2}}\right)\right. \\
& \left.+\frac{\left(k_{2}^{2}+k_{3}^{2}\right)}{k^{2}}\right\} \mathrm{d} A(\mathbf{k}), \\
E_{22}(\chi, t)= & \int_{|\boldsymbol{\chi}|=\chi} \frac{E(k, 0)\left(k_{1}^{2}+k_{3}^{2}\right)}{4 \pi \chi^{4}} \mathrm{~d} A(\mathbf{k}), \\
E_{33}(\chi, t)= & \int_{|\boldsymbol{\chi}|=\chi} \frac{E(k, 0)}{4 \pi k^{2}}\left\{\frac{k_{1}^{2} k_{3}^{2}}{k^{2}\left(k_{1}^{2}+k_{3}^{2}\right)}\left(\frac{-k^{2}}{k_{3}^{2}} a+\frac{k^{2}}{k_{1}^{2}} b\right)^{2}\right. \\
& \left.-\frac{2 k_{1} k_{2} k_{3}^{2}}{k^{2}\left(k_{1}^{2}+k_{2}^{2}\right)}\left(\frac{-k^{2}}{k_{3}^{2}} a+\frac{k^{2}}{k_{1}^{2}} b\right)+\frac{\left(k_{2}^{2}+k_{3}^{2}\right)}{k^{2}}\right\} \mathrm{d} A(\mathbf{k}),
\end{aligned}
$$

where,

$$
\begin{aligned}
a & =\frac{-k_{3}^{2}}{k_{1}\left(k_{1}^{2}+k_{2}^{2}\right)}\left\{\arctan \left(\frac{k_{2}}{\left(k_{1}^{2}+k_{3}^{2}\right)^{1 / 2}}\right)-\arctan \left(\frac{k_{2}-\beta k_{1}}{\left(k_{1}^{2}+k_{3}^{2}\right)^{1 / 2}}\right)\right\}, \\
b & =\beta k_{1}^{2}\left(k^{2}-2 k_{2}^{2}+\beta k_{1} k_{3}\right) / k^{2} \chi^{2} \\
k^{2} & =\chi_{1}^{2}+\left(\chi_{2}+\beta \chi_{1}\right)^{2}+\chi_{3}^{2} .
\end{aligned}
$$

We consider initial spectra of Gaussian and power law and forms,

$$
\begin{aligned}
& E(k, 0)=u_{0}^{2}(k L)^{N} \exp \left(-k^{2} L^{2}\right) \quad \text { where } N \text { is an integer and } N \geq 0, \\
& E(k, 0)=\frac{u_{0}^{2} L}{\left(1+k^{2} L^{2}\right)^{P}}, \quad \text { where } 1<P .
\end{aligned}
$$


The integrals for the $E_{i i}(\chi, t)$ can then be evaluated for $\beta \gg 1$ by asymptotic analysis. It is found that in the range $1 \ll \chi L \ll \beta E_{11}$ is much larger than the others, so $E(\chi, t) \approx E_{11}(\chi, t)$ and,

$$
E(\chi, t) \propto \frac{1}{k^{2}} \int_{|\chi|=\chi} \beta^{2} E(k L, 0) \mathrm{d} A(\mathbf{k}) .
$$

The major contribution to this integral comes from the region $|\hat{\theta}| \ll 1$ and $|\phi| \ll 1$, where $\hat{\theta}=\frac{1}{2} \pi+(1-\theta) / \beta$. So for $\beta \gg 1$ the integral (37) becomes,

$$
E(\chi, t) \propto \int_{-\infty}^{\infty} \int_{-\infty}^{\infty} \frac{\beta E\left(\left[\chi^{2} L^{2} / \beta^{2}+\chi^{2} L^{2}\left(\hat{\theta}^{2}+\phi^{2}\right)\right]^{1 / 2}\right)}{\chi^{2} L^{2}} \mathrm{~d}(\hat{\theta} \chi L) \mathrm{d}(\phi \chi L) .
$$

Thus, if $1 \ll \chi L \ll \beta$ if $E(k, 0)=\circ\left(k^{-2}\right)$ when $k L \ll 1$,

$$
E(\chi, t)=\beta\left(\int_{0}^{\infty} \hat{k} E(\hat{k}, 0) \mathrm{d} \hat{k}\right) / \chi^{2} L^{2}
$$

Therefore for large enough strain as long as the initial energy spectrum decreases faster than $k^{-2}$ the energy spectrum of sheared turbulence will tend to the limiting form of

$$
E(\chi, t) \propto \beta \chi^{-2}
$$

Note that this result will hold for a broader class of initial spectra than considered above, any spectrum with a falloff faster than $k^{-2}$ will suffice.

Figure 3 shows spectra from a number of experiments and simulations of shear flows. These results show a tendency towards a $\chi^{-2}$ region at large wavenumbers for large values shear. The result (39) means that also if the Reynolds number is moderate a shear flow will tend to maintain the $k^{-5 / 3}$ spectrum of very high Reynolds number turbulence, as has been observed in experiment (Monin \& Yaglom 1971 and reviewed by Hunt \& Vassilicos 1991).

This analysis has provided two very important pieces of information. First, it has shown that the form of the large wavenumber spectra in sheared turbulent flows (eg. turbulent boundary layers) may be determined by linear distortion by the mean shear, rather than by any complex nonlinear effects. Since $k^{-2}$ is close to $k^{-5 / 3}$ the spectra of shear flows are often discussed in terms of the spectral predictions of Kolmogorov theory which is based on a nonlinear cascade of energy to small scales! Secondly, the fact that the spectrum tends to the $\chi^{-2}$ form gives an indication of the type of structure one would expect in turbulent shear flows.

The energy spectrum, $E(\chi, t)$, of a function is proportional to the square of the modulus of the complex coefficients, $a_{n}$, of the Fourier series of $u(\mathbf{x})$,

$$
E(\chi, t) \propto\left|a_{n}(t)\right|^{2} \text { where } u(\mathbf{x})=\sum_{n} a_{n}(t) \exp \left(i \chi_{n}(t) x\right) .
$$

If $u(x)$ and all its derivatives are piecewise continuous then,

$$
\begin{gathered}
\left|a_{n}\right|=O\left(n^{-p}\right) \quad \text { as } \quad n \rightarrow \infty, \\
E(\chi, t)=O\left(\chi^{-2 p}\right) \quad \text { as } \quad \chi \rightarrow \infty,
\end{gathered}
$$


Figure 1: Schematic diagram showing how a spherical surface in the Fourier space of the local distorted wavenumber in a shear flow originates from a highly distorted region of wavenumber space at $t=0$. (a) Spherical shell in Fourier space at $t=T$, (b) shape of region in Fourier space of initial wavevectors which are mapped to the spherical shell by the shear distortion.

Figure 2: Schematic diagram of the change in energy spectra upon the application of strong shear, (a) before shear, (b) after shear. 
Figure 3: Changes in the energy spectra resulting from shear. (a) One dimensional spectrum measured by Champagne et al. (1970) in shear flow at two positions downstream. (b) $\chi^{2} E(\chi, t)$ and $\chi^{2} E_{i i}(\chi, t)$ in Direct Numerical Simulations of Rogallo (1981) of homogeneous turbulence in uniform shear $\left(R e_{\lambda} \approx 80\right)$. 
where $p-1$ is the lowest order continuous derivative (Courant \& Hilbert 1953, p. 73). Thus, if the velocity is discontinuous $E(\chi, t) \propto \chi^{-2}$ as $\chi \rightarrow \infty$, but the converse is not necessarily true. All that can be said is that if, as in a shear flow $E(\chi, t) \propto \chi^{-2}$, there will be singularities in the velocity, but not necessarily simple discontinuities. These singularities could also be an accumulating oscillation where the frequency of oscillation becomes singular (Hunt \& Vassilicos 1991). These singularities (actually sharp gradients when viscosity is included) in the velocity would be associated with elongated eddy structures, and just such 'stretched' eddies are seen in both DNS and experiments of shear flows.

\subsection{Turbulent structure}

RDT has been used to calculate the effect of uniform plane shear upon homogeneous, isotropic velocity field computed 'cheaply' using the method of Kinematic Simulation (KS) (Lee et al. 1990, Carruthers et al. 1991). In KS a turbulent-like 3-dimensional velocity field is generated from a truncated 3-dimensional Fourier series (usually about 38 terms is sufficient). The series is constructed so that the velocity field satisfies continuity. The magnitudes of the Fourier coefficients are chosen so that the velocity field has a prescribed energy spectrum, while the phases are chosen randomly from a set distributed isotropically on the unit sphere. KS produces accurate first and second order statistics (Fung et al. 1991) and allows quantities such as pressure and derivatives of velocity to be calculated exactly directly from the Fourier representation of the velocity. KS is also ideally suited for use with RDT since RDT calculates the evolution of Fourier coefficients. The velocity field produced using this combination of KS and RDT is shown in figure 4 below and compared with experimental, and Direct Numerical Simulation (DNS) results. Figure 4 shows good qualitative agreement between the RDT $+\mathrm{KS}$ results, the experiment and DNS. All three plots show extremely elongated eddy structures which lead to extreme gradients of velocity in the $y$-direction, consistent with the RDT analysis based on the asymptotic energy spectra of sheared turbulence. This is a case where the linear RDT analysis has captured all the essential dynamics, and has provided a simple structural explanation of the phenomenon.

\subsection{Perturbations to small scale high Reynolds number tur- bulence caused by the effect of shear and stratification}

In turbulent flows at very high Reynolds number, even if they are inhomogeneous and distorted, the small scales are approximately isotropic, as hypothesised in the universal equilibrium theory of Kolmogorov and as observed in many oceanic and atmospheric experiments (reviewed by Hunt \& Vassilicos 1991, Gibson 1991, Van Atta 1991). However, observations also show that there are systematic departures from isotropy that depend on the nature of the mean straining flow, the large scale motions and the effects of body forces. RDT can be applied to estimate these effects, because over a short enough time (defined later) the change caused by any mean strain or linear fluctuating body force on the fluctuating velocity field is larger than the changes caused by the nonlinear interactions of the turbulence (from the analysis in §3.2). 
Figure 4: Comparison of the effect of uniform shear upon turbulent flow, diagrams show contours of streamwise velocity. (a) Experiment on turbulent channel flow (Lee, Kim \& Moin 1990) $\beta=8, \alpha=35.1$, (b) Direct Numerical Simulation (Lee, Kim \& Moin 1990) $\beta=8, \alpha=33.6$, (c) RDT $+\mathrm{KS}, \beta=10, \alpha=50$, Von Karman energy spectrum, $E(k, 0)=1.196 k^{4} /\left(0.558+k^{2}\right)^{17 / 6}$. 
The novel aspect of this analysis is that we analyse a statistically steady process by performing an unsteady calculation of the changes to the turbulence over a certain range of length scales of order $k^{-1}$ that occur over a finite 'relaxation time scale' $\tau(k)$ whose magnitude depends on $k$. In high Reynolds number turbulence the relaxation time is determined by the nonlinear interactions between eddy motions on the same scale leading to $\tau(k) \sim \epsilon^{-1 / 3} k^{-2 / 3}$ whereas at low or moderate Reynolds numbers the large scale motions determine the relaxation time scale for all scales, $\tau(k) \sim L_{x} / u_{0}$. This approach is standard in statistical physics (for example in calculating the effect of polymers in fluids).

First consider the effect of mean shear on the high wavenumber spectrum. It follows from equations (15) to (20) that changes in the Fourier components when $t<\tau_{k}$ and when $k$ is large enough that $\tau_{k}$ is small (i.e. $k \gg \epsilon^{-1 / 2} \alpha^{3 / 2}$ ) are given by

$$
\begin{aligned}
& \hat{u}_{1}(\mathbf{k}, t)=\hat{u}_{1}(\mathbf{k}, 0)-\left(1-\frac{k_{1} k_{2}}{k^{2}}\right) \alpha t \hat{u}_{3}(\mathbf{k}, 0) \\
& \hat{u}_{2}(\mathbf{k}, t)=\hat{u}_{2}(\mathbf{k}, 0)-\frac{k_{1} k_{3}}{k^{2}} \alpha t \hat{u}_{3}(\mathbf{k}, 0) \\
& \hat{u}_{3}(\mathbf{k}, t)=\hat{u}_{3}(\mathbf{k}, 0)-\frac{k_{1} k_{2}}{k^{2}} \alpha t \hat{u}_{3}(\mathbf{k}, 0)
\end{aligned}
$$

Thence

$$
\frac{1}{2} \overline{\left(\hat{u}_{1}^{*} \hat{u}_{3}+\hat{u}_{1} \hat{u}_{3}^{*}\right)}=\Phi_{13}(\mathbf{k}, t)=-\left(\frac{k_{1} k_{3}}{k^{2}}+\left(1-\frac{k_{1}^{2}}{k^{2}}\right)\right) \alpha t \Phi_{33}(\mathbf{k})
$$

Assuming the turbulence is initially isotropic this leads to a change in Reynolds stress in a developing flow

$$
-\overline{u_{1} u_{3}}=2 / 5 \alpha \overline{u_{3}^{2}}(t=0)
$$

The one-dimensional cospectrum $\Theta_{13}(\mathbf{k}, t)$ can be obtained from (47) by substituting $\tau(k)$ to $t$ and integrating over $k$-space in the region $K_{1}$ (eg. $k=1 / 3 K_{1}$ to $3 K_{1}$ ) to obtain

$$
\Theta_{13}(\mathbf{k}, t)=C \alpha \epsilon^{2 / 3} K_{1}^{-7 / 3}
$$

where $C$ is of order 1.0. This result was first obtained experimentally, supported by physical and dimensional arguments by Wyngard \& Coté (1972). It has been rederived in several ways using approximate statistical models such as EDQNM (Bertoglio 1979). However, this analysis can be extended to derive the corrections to $\Theta_{11}, \Theta_{33}$; namely

$$
\begin{aligned}
\Theta_{11} & =\Theta_{11} \text { (isotropic) }\left(1-O\left(\alpha^{2} \tau^{2}\right)\right) \\
\Theta_{11} & =\alpha_{K} \epsilon^{2 / 3} K_{1}^{-5 / 3}-O\left(\alpha^{2} \epsilon K_{1}^{-3}\right) \\
\Theta_{33} & =\frac{4}{3} \alpha_{K} \epsilon^{2 / 3} K_{1}^{-5 / 3}-O\left(\alpha^{2} \epsilon K_{1}^{-3}\right)
\end{aligned}
$$

Note that the correction to the normal stress is much smaller than that to the Reynolds shear stress cospectra, which is consistent with the experiments.

Derbyshire \& Hunt (1992) have used the same approach to consider the combined effects of stable stratification and mean shear on the turbulence spectrum and thence 
explain why such large differences have been observed between the effects of stratification on low Reynolds number laboratory turbulence and on high Reynolds number atmospheric turbulence. From the study of Komori et al. (1983) and Hunt et al. (1988), the Reynolds shear stress and vertical variance in a developing shear flow with stable stratification determined by the buoyancy frequency $N$ are given by:

$$
\begin{aligned}
-\overline{u_{1} u_{3}} & =\frac{2}{5}(\alpha t) \overline{u_{3}^{2}}(t=0)-\frac{2}{15}(\alpha t) \overline{u_{3}^{2}}(t=0) t^{2} N^{2} \ldots, \\
\overline{u_{3}^{2}}(t) & =\left(1-\left(\frac{4}{35} \alpha^{2}+\frac{4}{5} N^{2} t^{2}\right)\right) \overline{u_{3}^{2}}(t=0) .
\end{aligned}
$$

Using the same argument as in (50) the correction of the spectrum caused by stable stratification can be found by including both the RDT shear and buoyancy terms of (53),

$$
\Theta_{13}\left(K_{1}\right) \sim-\Theta_{33} \alpha \epsilon^{-1 / 3} K_{1}^{-2 / 3}\left(1-O\left(\epsilon^{-2 / 3} K_{1}^{-4 / 3} N^{2}\right)\right)
$$

Now, from (54) the shear and buoyancy correction to the isotropic value of $\Theta_{33}\left(K_{1}\right)$ is,

$$
\Theta_{33}\left(K_{1}\right) \sim \frac{4}{3} \alpha_{K} \epsilon^{2 / 3} K_{1}^{-5 / 3}\left[1-O\left(\epsilon^{-2 / 3} K_{1}^{-4 / 3}\left(\alpha^{2}+N^{2}\right)\right)\right],
$$

which is of the same order as the correction to $\Theta_{13}$. Here $\alpha_{K}$ is the Kolmogorov constant $\simeq 1.5$.

A different analysis is necessary for low Reynolds number turbulence whose strain spectrum $\left(\propto K_{1}^{2} \Theta_{13}\left(K_{1}\right)\right)$ decreases with $K_{1}$ in this range. In this case the largest strain rate and therefore the characteristic time scale $\tau(k)$ is determined by the large scales and not the small scales, i.e. $\tau(K)\left(\sim L / u_{0}\right)$ is independent of $K$ where $u=0$ is the rms turbulence. Then from (53) as the Richardson number increases $\left|\Theta_{13}\left(K_{1}\right)\right|$ decreases over the whole range of $K_{1}$, ie.,

$$
\Theta_{13}\left(K_{1}\right) \sim \Theta_{13}\left(K_{1}\right)(R i=0)-O\left[\left(L / u_{0}\right)^{3} \alpha N^{2}\right] \Theta_{33}\left(K_{1}\right)
$$

Comparing (55) and (57) shows that since the corrections for high Reynolds number in (55) are small for $R i \lesssim 0.2$, the shear stress ratio $\left(-\overline{u_{1} u_{3}}\right) / u_{3}^{2}$ is expected to vary slowly for high Reynolds number turbulence over this range of $R i$. The low Reynolds number correction in (54), however, is larger than the respective high Reynolds number correction and so the shear stress ratio decreases significantly in the typical 'low' Reynolds number turbulence found in the laboratory and computed with D.N.S. (eg. Van Atta 1991). To the extent that stable stratification does affect the high wavenumber spectrum in high Reynolds number turbulence, the greater correction to $\Theta_{13}$ in (55) than to $\Theta_{33}$ in (56) explains why the shear stress ratio decreases when $R i \gtrsim 0.2$.

Thus we have seen that in moderately stratified turbulent flows at high Reynolds numbers (with $R i \lesssim 0.25$ ) the structure of the turbulent velocity field (eg. the shear stress ratio) is controlled by shear, although the velocity profiles and statistics will vary. We have also seen that under certain conditions (i.e. $t \mathrm{~d} U / \mathrm{d} z \sim 4$ and $R i \sim 0.25$ ) the effects of shear and buoyancy effectively cancel and the shear stress $\overline{u w}$ becomes zero. Note that in this case the structure of density fluctuations are controlled primarily by density forces.

This is another example of how RDT analysis has given insight into the structure of turbulent flows using simple hypotheses, and analytical methods. 


\section{Structures and dynamics in distorted turbulence}

In this section we model the interaction between the large-scales and small-scales of turbulence as a rapid distortion of the small-scales by the large-scales. The results presented include changes in dynamical quantities and a structural analysis.

Three-dimensional homogeneous, isotropic turbulence generated using KS is subjected to combinations of rotational and irrotational plane distortions ranging from pure rotation through pure shear to pure irrotational strain. The type of distortion experienced by an eddy is, in practice, related to the type of large-scale structure which is distorting the small-scales. For example, a large-scale eddy would induce a rotational distortion, whereas the regions between eddies would induce irrotational distortions and an intermediate position around an eddy would usually lead to a combination of these effects, i.e. a shear distortion. The picture of turbulence we put forward is one where the small-scale eddies are advected past the large-scale eddies thereby passing through regions producing a variety of distortions. The effect of these distortions was calculated using RDT. For arbitrary combinations of rotational and irrotational distortion the RDT equations cannot be solved analytically for general initial conditions, so the equations were solved numerically using an adjustable step-size method. A variety of dynamical quantities (eg. vorticity production, alignment of eigenvectors of rate of strain with vorticity, angle between velocity and vorticity) were measured before and after the distortions.

The purpose of this investigation is to determine which aspects of the dynamics of turbulence can be accounted for by the simple linear dynamics of RDT. Or, more specifically, what aspects of 'real' turbulence that are not seen in random or Gaussian turbulence may be seen when Gaussian turbulence (generated by KS) is subjected to a rapid distortion? An important case where the origin of a dynamical effect is unclear is the alignment of the middle eigenvector (associated with the middle eigenvalue) of the rate of strain tensor with the vorticity. This alignment is not seen in Gaussian or random turbulence (Shtilman et al. 1992). Ashurst et al. (1987) and Vincent \& Meneguzzi (1991) have observed such an alignment in Direct Numerical Simulations and believed it to arise from poorly understood nonlinear effects.

The flow is also divided into different structure types using an algorithm we developed based on values of pressure, speed and the parameter $\Sigma$ defined as,

$$
\Sigma=\frac{S_{i j}^{2}-\Omega_{i j}^{2}}{S_{i j}^{2}+\Omega_{i j}^{2}},
$$

where $S_{i j}$ is the rate of strain tensor, and $\Omega_{i j}$ is the vorticity tensor. The five basic structure types are: eddy $-\Sigma<-1 / 3$ and $p<0$; donor eddies $-\Sigma<-1 / 3$ and $p>0$; shear- $-1 / 3 \leq \Sigma \leq 1 / 3$; convergence $-\Sigma>1 / 3$ and $p>0$; and streaming$|u|>u_{\mathrm{RMS}}$ and $|\Omega|<\frac{1}{2}|\Omega|_{\mathrm{RMS}},|S|<\frac{1}{2}|S|_{\mathrm{RMS}}$. (Note that for all regions except streams the level of strain must be above a certain threshold, i.e. $|\Omega| \geq \frac{1}{2}|\Omega|_{\text {RMS }}$, $|S| \geq \frac{1}{2}|S|_{\text {RMS }}$.) Thus eddies are regions of high vorticity with closed streamlines about a low pressure core; donor eddies are regions of high vorticity and positive pressure fluctuation without closed streamlines, shear structures are regions of shear straining; convergence structures are regions of irrotational straining characterised by converging 
Figure 5: Schematic diagram of the five structure types. (a) Eddy, (b) donor eddy, (c) shear, (d) convergence, (e) streaming.

streamlines and positive pressure fluctuation; and stream structures are regions of low deformation and high kinetic energy. This algorithm identifies about $80 \%$ of the initial flow. This approach differs from that suggested by Wray \& Hunt (1990) in that the conditions are absolute, based on the dimensionless parameter $\Sigma$ (apart from a lower limit of $1 / 2$ RMS values of $S$ and $\Omega$ ) which means that similar regions of different flows will be identified as the same structure type; donor regions are recognized; the two cases of small second invariant are distinguished (i.e. shear when the $S$ and $\Omega$ are both large, but approximately equal, and unstructured or stream when $S$ and $\Omega$ are both small). These differences mean that the identifications correspond well with a qualitative visual classification of the flow, and that about $50 \%$ more of the flow is classified than in the Wray \& Hunt (1990) algorithm.

Dividing a flow into different structures is a way of characterizing the flow. Each structure has specific properties (eg. eddies trap particles, convergence region increase the efficiency of mixing) and by seeing how the number of these regions changes with the type of applied distortion one can understand how the overall properties of the flow changes.

Jets of turbulent water are sometimes used to control dust, and turbulence is often used to accelerate mixing. If the types of distortions that increase eddy and convergence regions can be found then these distortions could be applied to increase the efficiency of the working fluids in dust control and mixing respectively.

Distortions with large-scale $S+\Omega=20.0$ and $t=0.1$ (giving a ' $\beta$ ' of 2.0 ) were applied to initial KS flow with both the Von Karman spectra,

$$
E(k, 0)=1.196 k^{4} /\left(0.558+k^{2}\right)^{17 / 6},
$$

and exponential spectra,

$$
E(k, 0)=k^{2} \exp \left(-0.75 k^{2}\right) .
$$


Figure 6: PDF showing the alignment of vorticity with the intermediate eigenvector of the small-scale rate of strain tensor. (a) Before distortion (Gaussian profile), (b) after distortion, $\left(S^{L-S}-\Omega^{L-S}\right) /\left(S^{L-S}+\Omega^{L-S}\right)=0.8, t=0.10$.

It was found that the vorticity aligned with the intermediate eigenvector of the smallscale rate of strain matrix, but with the largest eigenvector of the large-scale (applied) strain (see figure 6). This alignment increased with the proportion of irrotational straining in the applied distortion. The alignment with the largest eigenvector of the applied strain is clearly expected from an examination of the vorticity equation,

$$
\frac{D \boldsymbol{\omega}}{D t}=\boldsymbol{\omega} \cdot\left(\mathbf{S}^{S-S}+\mathbf{S}^{L-S}\right)-(\mathbf{U} \cdot \nabla) \boldsymbol{\omega},
$$

where $\mathbf{S}^{S-S}$ is the small-scale rate of strain tensor and $\mathbf{S}^{L-S}$ is the large-scale rate of strain tensor. The less obvious alignment with the small-scale rate of strain tensor can be shown to increase exponentially with time using an RDT analysis for large time.

This result shows that the alignment of vorticity with the local intermediate eigenvector of the rate of strain observed in DNS is not caused by any particular nonlinear effects, but largely arises simply from irrotational straining. This irrotational straining also tends to produce the long 'worm-like' vortex tubes containing most of the high intensity vorticity also seen in DNS (Vincent \& Meneguzzi 1991). Thus, irrotational straining could lead both to the creation of long, thin vortex tubes and associated with this, the alignment between vorticity and the intermediate eigenvector of the rate of strain tensor.

Local vorticity production was measured by calculating $\omega_{i} \omega_{j} S_{i j}$ which is the source term in the enstrophy equation,

$$
\frac{D\left(\frac{1}{2} \omega^{2}\right)}{D t}=\omega_{i} \omega_{j} S_{i j}-\nu\left(\frac{\partial \omega_{i}}{\partial x_{j}}\right)^{2} .
$$


Figure 7: PDF's of vorticity production, $\omega_{i} \omega_{j} S_{i j}$. (a) Initially (Gaussian profile), (b) after large-scale irrotational distortion $\left(\left(S^{L-S}-\Omega^{L-S}\right) /\left(S^{L-S}+\Omega^{L-S}\right)=0.8, t=0.10\right.$.

Initially there is no net vorticity production (Gaussian PDF), and no distortion produced any overall vorticity production. It is found, however, that vorticity production is dramatically increased under high applied irrotational distortion in regions with a positive intermediate eigenvalue (note that because of incompressibility the sum of the eigenvalues is zero) of the small scale rate of strain tensor, and vorticity was destroyed in regions with a negative intermediate eigenvalue. This result was essentially predicted by Betchov (1956).

Figure 7 shows the positively skewed PDF of $\omega_{i} \omega_{j} S_{i j}$ (indicating net production of vorticity) under high applied irrotational strain in these 'Betchov' regions. It can be shown using an RDT calculation for large times that this production (or destruction) becomes exponentially large with time under irrotational strain,

$$
\left\langle\omega_{i} \omega_{j} S_{i j}\right\rangle=\left\langle b \omega_{10}^{2}\right\rangle \exp \left(2 S^{L-S} t\right) \text { as } t \rightarrow \infty,
$$

where $S^{L-S}$ is the large-scale strain, $\omega_{10}$ is the initial velocity in the direction of the positive eigenvector of applied strain, and $b$ is the intermediate small-scale eigenvalue. This destruction of vorticity in some strained regions and its production in other strained regions (depending on local values of the eigenvalues of the rate of strain tensor) will eventually lead to highly intermittent vorticity. Thus, in a turbulent flow where the vorticity is initially evenly distributed, linear straining effects will tend to lead to the concentration of vorticity in small regions- -thin vortex tubes. Again, we see linear effects largely responsible for a fundamental aspect of turbulence, the highly intermittent distribution of vorticity.

The alignment between velocity and vorticity retained its Gaussian PDF for all distortions and within all structure types. The number of 'cigar' regions (negative intermediate eigenvalue) and 'pancake' (positive intermediate eigenvalue) regions remained roughly constant at $50 \%$ each under all distortions. 
Figure 8: Percentage change in number of structures as a function of type of applied distortion. $\Sigma=(S-\Omega) /(S+\Omega)$. (a) Eddies, (b) donor eddies, (c) convergence regions, (d) shear regions, (e) stream regions.

The change in the relative proportion of each of the structures with different types of large-scale distortion is shown in figure 8 . Figure 8 shows that the percentage of eddies, convergence and shear regions increased with the proportion of rotational distortion, while the percentage of streams and donor eddies increased with proportion of irrotational distortion. The average percentage of eddies over the range of distortions about equals the initial value, while convergence, shear and donor regions are suppressed relative to the initial amount for all, but high rotational distortion. Stream regions, on the other hand, are increased relative to the initial value for all distortions except at high rotational distortion. An interesting point is that for eddies and shear regions a pure shear distortion does not change their number. Changes in percentage of convergence regions mirrors the changes in eddy regions. This is expected since convergence regions are associated with the area between eddies. Shear regions underwent the greatest overall change $(30 \%)$. These results indicate one should apply rotational distortion to the turbulence used in dust curtains and mixing fluids!

All results in this section except the change in the percentage of the different structure types were independent of the energy spectrum (Von Karman or exponential) used. The changes in the number of structure types with applied distortion was significantly less, but qualitatively the same, for the Von Karman spectrum. There is much more energy in the large wavenumber part of the spectrum in the Von Karman spectrum, indicating most of the structural effects are due to changes in the small-wavenumber (large-scale) part of the spectrum. 


\section{Conclusions}

As explained in the introduction and in $\S 2$ Rapid Distortion Theory is formally a method of calculating turbulent flows changing from a defined state under the influence of a linear distortion that has a stronger effect on the inertial term than on the nonlinear interactions of the turbulence. Most of the literature of RDT is concerned with such problems, which may be quite complex in inhomogeneous distortion, boundaries, etc.

However, starting with studies of Townsend (1976), it has long been recognised that this linear theory also gives some insight into the structure of turbulence even when it is slowly changing and approximately independent of initial or boundary conditions. This is the aspect of RDT that has been emphasised in this review. RDT has demonstrated how, only if the mean flow is approximately like a shear flow, do the energy containing eddies become approximately independent of their initial conditions and that the turbulence tends to a constant form of self-similar spectrum. We have not proved that the linear theory demonstrates this property, the nonlinear turbulence must also exhibit the same property; but it is likely on physical grounds and it is observed. RDT has also provided a straight forward method for estimating the corrections to isotropic high Reynolds number turbulence for different kinds of distortion. This approach could be developed further with more detailed calculation and different hypotheses about the relaxation. More detailed comparison with other models such as EDQNM could be interesting. The results are sufficiently interesting to stimulate new experimental measurement.

Thirdly, RDT now provides an interesting method for simulating how the eddy structure of turbulence evolves both under the action of mean distortion, (following Lee et al. 1990), and even during the mutual random interaction of eddy motion between different scales. The preliminary results presented here show that this kind of interaction explains some of the salient nonlinear processes in fully developed turbulence and indicates how different types of eddy motion evolve. Clearly further analysis in this direction is possible.

\section{Acknowledgements}

N. Kevlahan was supported during this research by a British Government ORS award and a British Gas research grant.

\section{References}

Adrian, R.J. \& Moin, P. 1988. Stochastic estimation of organized turbulent structure: homogeneous shear flow. J. Fluid Mech., 190, 531-559.

Ashurst, W.T., Kerstein, A.R., Kerr, R.M. \& Gibson, C.H. 1987. Alignment of vorticity and scalar gradients with strain rate in simulated Navier-Stokes turbulence. Phys. Fluids 30(8), 2343-2353.

Barcilon, A., Brundley, J., Lessan, M. \& Mobbs, F.R. 1979. Marginal instability in 
Taylor-Couette flows at a very high Taylor number. J. Fluid Mech. 94, 453-463.

Batchelor, G.K. 1953. Homogeneous turbulence. Cambridge University Press.

Batchelor, G.K. 1955. The effective pressure exerted by a gas in turbulent motion. In Vistas in Astronomy (ed. A. Beer) 1, 290-295. Pergamon.

Batchelor, G.K. \& Proudman, I. 1954. The effect of rapid distortion of a fluid in turbulent motion. J. Mech. \&5 App. Math. 7, pt. 1, 83-103.

Betchov, R. 1956. An inequality concerning the production of vorticity in isotropic turbulence. J. Fluid Mech. 1, 497-504.

Bevilaqua, P.M. \& Lykoudis, P.S. 1978. Turbulence memory in self-preserving wakes. J. Fluid Mech. 89, 589-606.

Cambon, C. 1992. Ercoftac Workshop Proceedings. To appear.

Carruthers, D.J., Fung, J.C.H., Hunt, J.C.R., Perkins, R.J. 1991. The emergence of characteristic (coherent?) motion in homogeneous turbulent flows. In Turbulence and coherent structures (eds. M. Lesieur \& O. Métais), pp. 29-44. Kluwer.

Champagne, F.H., Harris, V.G. \& Corrsin, S. 1970. Experiments on nearly homogeneous turbulent shear flow. J. Fluid Mech. 41, 81-139.

Courant, R. \& Hilbert, D. 1953. Methods of mathematical physics, 1. New York: Interscience.

Craik, A.D.D. \& Criminale, W.O. 1986. Evolution of wavelike disturbances in shear flows: a class of exact solutions of the Navier-Stokes equations. Proc. R. Soc. Lond. A. 406, 13-26.

Derbyshire, S.H. \& Hunt, J.C.R. 1992. Structure of turbulence in stably stratified atmospheric boundary layers; comparison of large eddy simulations and theoretical models. In Proc. $3^{\text {rd }}$ I.M.A. Conf. on Stably Stratified Flows, 'Waves and Turbulence in Stably Stratified Flows' (ed. S.D. Mobbs). Clarendon Press: Oxford.

Durbin, P.A. \& Zeman, O. 1991. Rapid distortion theory for homogeneous turbulence with application to modeling. Submitted to J. Fluid. Mech.

Falco, R.E. 1991. A coherent structure model of the turbulent boundary layer and its ability to predict Reynolds number dependence. Phil. Trans. R. Soc. Lond. A. 336, 103-129.

Frisch, U. 1991. From global scaling à la Kolmogorov, to local, multifractal scaling in fully developed turbulence. Proc. R. Soc. Lond. A. 434, 89-99.

Fung, J.C.H., Hunt, J.C.R., Malik, N.A. \& Perkins, R.J. 1991. Kinematic simulation of homogeneous turbulent flows generated by unsteady random Fourier modes. J. Fluid 
Mech. (In the press.)

Gibson, C.H. 1991. Kolmogorov similarity hypotheses for scalar fields: sampling intermittent turbulent mixing in the ocean and galaxy. Proc. R. Soc. Lond. A. 434, $149-164$

Goldstein, M.E. 1978. Unsteady vortical and entropic distortion of potential flow round arbitrary obstacles. J. Fluid Mech. 89, 433-468.

Hunt, J.C.R. 1973. A theory of turbulent flow round two-dimensional bluff bodies. J. Fluid Mech. 61, 625-706.

Hunt, J.C.R. 1992a. Developments in computational modelling of turbulent flows. ERCOFTAC workshop introductory lecture. To appear.

Hunt, J.C.R. 1992b. Rapid Distortion Theory. Lecture notes for the ERCOFTAC Summer School. Cambridge University Press.

Hunt, J.C.R. \& Carruthers, D.J. 1990. Rapid distortion theory and the 'problems' of turbulence. J. Fluid Mech., 212, 4997-532.

Hunt, J.C.R., Stretch, D.D. \& Britter, R.E. 1988. Length scales in stably stratified turbulent flows and their use in turbulence models. In Stably stratified flow and dense gas dispersion ( ed. J.S. Puttock). Oxford: Clarendon Press.

Hunt, J.C.R. \& Vassilicos, J.C. 1991. Kolmogorov's contributions to the physical and geometrical understanding of small-scale turbulence and recent developments. Proc. R. Soc. Lond. A, 434, 183-210.

Hussain, A.K.M.F. 1986. Coherent structures and turbulence. J. Fluid Mech. 173, 303-356.

Kaltenbach, D.-J., Gertz, T. \& Schumann, U. 1989. Transport of passive scalars in neutrally and stably stratified homogeneous turbulent shear flows. In Proc. 7th shear flows conference. Springer.

Kelvin, Lord (W. Thomson) 1887. Stability of fluid motion: rectilinear motion of viscous fluid between two parallel plates. Phil. Mag. 24(5), 188-196.

Kida, S. \& Hunt, J.C.R. 1989. Interaction between different scales of turbulence over short times. J. Fluid Mech. 201, 411-445.

Kolmogorov, A.N. 1941. The local structure of turbulence in incompressible viscous fluid for very large Reynolds numbers. Dokl. Akad. Nauk SSSR, 30(4), p. 301.

Komori, S., Ueda, H., Ogino, F. \& Mizushina, T. 1983. Turbulence structure in stably stratified open-channel flow. J. Fluid Mech. 130, pp. 13-26.

Lee, M.J., Kim, J. \& Moin, P. 1990. The structure of turbulence at high shear rate. 
J. Fluid Mech. 216, 561-583.

Lumley, J. 1965. The structure of inhomogeneous turbulent flows. Proc. Intl. Coll. on Radio Wave Propagation (ed. A.M. Yaglom \& V.I. Takasky), Dokl. Akad. Nauk. SSSR, 166-178.

Monin, A.S. \& A.M. Yaglom. 1971. Statistical theory of turbulence, II. MIT Press.

Mumford, J.C. 1982. The structure of the large eddies in fully developed turbulent shear flows. Part 1. The plane jet. J. Fluid Mech. 118, 241-268.

Reynolds, W.C. 1980. Symp. Combustion Model Recip. Eng. New York: Plenum Press, 41-66.

Rogallo, R.S. 1981. Numerical experiments in homogeneous turbulence. NASA Tech. Memo., 81315.

Shtilman, L., Spector, M. \& Tsinober, A. 1992. On some kinematic versus dynamic properties of homogeneous turbulence. (submitted to J. Fluid Mech.)

Taylor, G.I. 1935. Turbulence in a contracting stream. Z. angew. Math. Mech., 15, p. 91.

Townsend, A.A. 1958. Turbulent flow in a stably stratified atmosphere. J. Fluid Mech. 3, 361-372.

Townsend, A.A. 1976. The structure of turbulent shear flow. Cambridge University Press.

Tucker, H.J. \& Reynolds, A.J. 1968. The distortion of turbulence by irrotational plane strain. J. Fluid Mech. 32, 657-673.

Van Atta, C. 1991. Local isotropy of the smallest scales of turbulent scalar and velocity fields. Proc. R. Soc. Lond. A. 434, 139-148.

Vincent, A. \& Meneguzzi, M. 1991. The spatial structure and statistical properties of homogeneous turbulence. J. Fluid Mech. 225, 1-20.

Wray, A.A. \& Hunt, J.C.R. 1990. Algorithms for classification of turbulent structures. In Proc. IUTAM Symp. 1989, Topological fluid mechanics (eds. H.K. Moffatt and A. Tsinober), 95-104. Cambridge University Press.

Wyngaard, J.C. \& Coté, O.R. 1972. Modelling buoyancy driven mixed layers. J. Atmos. Sci. 33, 1974-1988. 


\section{Addresses of the authors}

Prof. J.C.R. Hunt

Meteorological Office

London Road

Bracknell

Berkshire

U.K. RG12 2SZ

N. Kevlahan

University of Cambridge

Dept. of Applied Maths. and Theoretical Physics

Silver St.

Cambridge

U.K. CB3 9EW 